\title{
Efficacy of nicorandil treatment for prevention of contrast-induced nephropathy in high-risk patients undergoing cardiac catheterization: A prospective randomized controlled trial
}

Leili Iranirad ${ }^{1}$, Seyed Fakhreddin Hejazi ${ }^{1}$, Mohammad Saleh Sadeghi ${ }^{2,3}$, Shahram Arsang Jang ${ }^{4}$

${ }^{1}$ Department of Cardiology, Qom University of Medical Sciences, Qom, Iran ${ }^{2}$ Student Research Committee, Qom University of Medical Sciences, Qom, Iran

${ }^{3}$ School of Medicine, Zahedan University of Medical Sciences, Zahedan, Iran

${ }^{4}$ Department of Biostatistics and Epidemiology, Karmanshah University of Medical Sciences, Karmanshah, Iran

\begin{abstract}
Background: Contrast-induced nephropathy (CIN) remains to be a potentially serious complication of radiographic procedures and is the third leading cause of the acute kidney injury (AKI) among hospitalized patients. This clinical trial was performed to assess the preventive effect of oral nicorandil on CIN in high-risk patients undergoing cardiac catheterization.

Methods: In this prospective, randomized, controlled trial, 128 patients with at least two risk factors for CIN undergoing elective percutaneous coronary intervention (PCI) were randomly assigned to either the nicorandil group or the control group. Patients in the nicorandil group $(n=64)$ received $10 \mathrm{mg}$ nicorandil, daily from $30 \mathrm{~min}$ before and up to 3 days after procedure and intravenous hydration for $2 h$ before and $6 h$ after the procedure, whereas patients in the control group $(n=64)$ just received intravenous hydration. Serum creatinine ( $\mathrm{SCr}$ ) was measured before contrast exposure and at $72 \mathrm{~h}$. CIN was defined as an increase of $25 \%$ in SCr or $>0.5 \mathrm{mg} / \mathrm{dL} 72 \mathrm{~h}$ after contrast administration.

Results: Contrast-induced nephropathy occurred in 14 out of 64 (21.9\%) patients in the control group and in 3 out of 64 (4.7\%) patients in the nicorandil group. There was a significant difference in the incidence of CIN between the two groups at $72 \mathrm{~h}$ after administering the radiocontrast agent $(p=0.008)$. Moreover, there were significant differences between the two groups in $\mathrm{SCr}$ and estimated glomerular filtration rate $72 h$ after radiocontrast administration $(p<0.05)$.

Conclusions: The findings revealed that oral nicorandil had substantial efficacy over hydration protocol for the development of CIN in high-risk patients undergoing cardiac catheterization. (Cardiol J 2017; 24, 5: 502-507)
\end{abstract}

Key words: contrast-induced nephropathy, nicorandil, percutaneous coronary intervention

\section{Introduction}

Contrast-induced nephropathy (CIN), also known as contrast-induced acute kidney injury (CI-AKI) is a well-known, prevalent and serious side effect of the administration of iodine contrast medium after angiocardiography or radiological procedures $[1,2]$. In clinical studies, $\mathrm{CIN}$ is defined as an elevation of serum creatinine level $44.2 \mu \mathrm{mol} / \mathrm{L}$ $(0.5 \mathrm{mg} / \mathrm{dL})$ or $25 \%$ above the baseline within 48-72 $\mathrm{h}$ after contrast administration without an alternative cause [3-5]. The exact pathophysiological mechanisms of CIN are complex and remain unclear. CIN incidence is increasing, ranging from

Address for correspondence: Mohammad Saleh Sadeghi, MD, Student Research Committee, Qom University of Medical Sciences, Qom, Iran, tel: +98 91767830 34, e-mail: salehsadeghi87@gmail.com 
$2 \%$ in the general population to $50 \%$ in high-risk patients with conditions like chronic renal impairment or having certain risk factors $[2,3,6]$. The most critical risk factors for CIN include diabetes mellitus (which is associated with increased risk even in patients with preserved renal function), congestive heart failure (CHF), age $>75$, hypertension, hypotension, decreased renal perfusion, female gender, high-osmolar contrast, contrast volume, urgent vs. planned PCI and most importantly, chronic kidney disease (CKD) $[7,8]$. CIN is the third leading cause of acute kidney injury (AKI) in hospitalized patients, accounting for more than $10 \%$ of all renal failure patients and is also associated with increased risk of CKD progression and dialysis, increased health care costs, increased morbidity and mortality $[3,9,10]$. Therefore, preventive measures for CIN are inevitable and remains a challenge among cardiologists and radiologists.

Nicorandil is a K-ATP channel agonist and nitric oxide (NO) donor that have been used to treat angina and heart failure. Recent studies revealed that nicorandil may protect the kidney from ischemic injury associated with the use of contrast media by ameliorating ischemic preconditioning [11-13]. Nevertheless, few studies show the effects of nicorandil in CIN. In the present study, it was sought to demonstrate the efficacy of oral administration of nicorandil for CIN prevention in a prospective, randomized trial in high-risk patients undergoing elective PCI.

\section{Methods}

The protocol for this study was approved by the Ethics Committee of Qom University of Medical Sciences (approval number: IR.MUQ. REC.1394.134), and the written informed consent was obtained from all patients before enrollment. This study was conducted in accordance with the principles of the Declaration of Helsinki.

\section{Study population}

This study was a prospective, open-label, randomized controlled trial. All adult ( $>18$ years) patients scheduled for PCI were screened for inclusion and exclusion criteria. The inclusion criteria were the existence of at least two of the following CIN risk factors (at least moderate risk for CIN as defined by Mehran risk score) [14]: systolic heart failure (with documented ejection fraction $<40 \%$ ), hypertension and diabetes mellitus (noted in their past medical history), age $>75$ years and renal insufficiency (estimated glomerular filtration rate
[eGFR] $<60 \mathrm{~mL} / \mathrm{min} / 1.73 \mathrm{~m}^{2}$, or baseline serum creatinine $[\mathrm{SCr}]>1.5 \mathrm{mg} / \mathrm{dL}$ ). The exclusion criteria included: end-stage renal insufficiency (eGFR $<15 \mathrm{~mL} / \mathrm{min}$ ), acute renal insufficiency, pregnancy and lactation, pulmonary edema, cardiogenic shock, multiple myeloma, history of an allergic reaction to contrast agents or nicorandil, contrast media exposure within 7 days before the procedure, uremia, renal failure which led to having dialysis, and the administration of $\mathrm{N}$-acetyl cysteine (NAC), metformin, dopamine, theophylline, sodium bicarbonate, mannitol, fenoldopam, diuretics, and nephrotoxic medicines within $48 \mathrm{~h}$ before the procedure.

Each patient randomly received an individual number which accordingly divided them into two groups; odd numbered patients were assigned to group 1 (nicorandil group) and even numbered to group 2 (control group).

\section{Study protocol}

A total of 128 eligible patients were randomly assigned to either the nicorandil group $(n=64)$ or the control group $(n=64)$, using a balanced block randomization method. Patients in the nicorandil group received $10 \mathrm{mg}$ nicorandil, daily from $30 \mathrm{~min}$ before to 3 days after the procedure and standard intravenous hydration $(1 \mathrm{~mL} / \mathrm{kg} / \mathrm{h})$ via normal saline, a maximum $100 \mathrm{~mL} / \mathrm{h}$ for $2 \mathrm{~h}$ before and $6 \mathrm{~h}$ after the procedure, whereas patients in the control group received intravenous hydration via the same method. SCr was measured before contrast exposure and at $72 \mathrm{~h}$ after it.

Several parameters were analyzed in the overall population. The GFR was estimated using the Cockcroft-Gault formula, $(140-$ age $) \times$ weight $[\mathrm{kg}] /(\mathrm{SCr} \times 72)$ in male patients with adjustment for female patients multiplied by 0.85 . The kidney function was categorized according to the stages set by the United States National Kidney Foundation and defined by the eGFR value as follows: normal kidney function: GFR $\geq 90 \mathrm{~mL} / \mathrm{min}$ and no proteinuria; mild kidney damage: GFR of 60 $-89 \mathrm{~mL} / \mathrm{min}$, with evidence of kidney damage; moderate damage: GFR of $30-59 \mathrm{~mL} / \mathrm{min}$; severe damage: GFR of $15-29 \mathrm{~mL} / \mathrm{min}$; and kidney failure (dialysis): GFR $<15 \mathrm{~mL} / \mathrm{min} / 1.73 \mathrm{~m}^{2}$.

All the tests were performed in the same laboratory with the same methodology. PCIs were performed through the femoral artery with the low osmolar nonionic contrast agent iohexol (Omnipaque, GE Healthcare, Cork, Ireland). Echocardiographic evaluations of all patients before exposure were also performed. 
Table 1. Baseline clinical and procedural characteristics of the patients.

\begin{tabular}{lccc}
\hline Variables [mean \pm SD or $\mathbf{n}(\%)$ ] & Nicorandil group & Control group & $P^{*}$ \\
\hline Male & $39(60.9 \%)$ & $40(62.5 \%)$ & 0.856 \\
Age [year] & $61.35 \pm 11.77$ & $57.64 \pm 12.42$ & 0.085 \\
Age $>75$ years & $10(15.6 \%)$ & $8(12.5 \%)$ & 0.611 \\
Systemic hypertension & $35(54.7 \%)$ & $41(64.1 \%)$ & 0.280 \\
Diabetes mellitus & $27(42.2 \%)$ & $26(40.6 \%)$ & 0.858 \\
Smoking & $22(36.7 \%)$ & $13(31 \%)$ & 0.550 \\
Hemoglobin & $13.51 \pm 1.5$ & $14.8 \pm 3.6$ & 0.010 \\
Hematocrit & $41.35 \pm 4.6$ & $42.60 \pm 6.1$ & 0.194 \\
Fasting blood sugar & $133.62 \pm 56.7$ & $132.53 \pm 56.1$ & 0.913 \\
Triglyceride & $153.92 \pm 70$ & $165.59 \pm 86.5$ & 0.403 \\
HDL & $42.98 \pm 10.8$ & $42.31 \pm 11$ & 0.730 \\
LDL & $91.5 \pm 38.1$ & $100.98 \pm 42.4$ & 0.186 \\
BMI $\left[\mathrm{kg} / \mathrm{m}^{2}\right.$ ] & $28.43 \pm 5.6$ & $27.78 \pm 4.8$ & 0.484 \\
LVEF [\%] & $48.87 \pm 6.8$ & $49.14 \pm 5.8$ & 0.812 \\
LVEF < $40[\%]$ & $9(14.5 \%)$ & $11(17.2 \%)$ & 0.682 \\
GFR < 60 [mL/min/1.73 $\left.\mathrm{m}^{2}\right]$ & $19(29.7 \%)$ & $13(20.3 \%)$ & 0.221 \\
Dose of contrast agent $\left[\mathrm{mL}^{2}\right.$ & $213.98 \pm 44.6$ & $202.26 \pm 44.4$ & 0.139 \\
\hline
\end{tabular}

*Between-group comparisons were assessed using independent samples t-test for the normally distributed value or the Mann-Whitney $U$ for non-normally distributed values. The categorical variables were compared through using Pearson chi-square test; HDL - high-density lipoprotein; LDL — low-density lipoprotein; BMI — body mass index; LVEF — left ventricular ejection fraction; GFR — glomerular filtration rate

\section{Study endpoints}

The primary endpoint of the study was the development of $\mathrm{CIN}$, which is defined as an increase in $\mathrm{SCr}$ level at $44.2 \mu \mathrm{mol} / \mathrm{L}(0.5 \mathrm{mg} / \mathrm{dL})$ or $25 \%$ above the baseline within $72 \mathrm{~h}$ after contrast medium administration without an alternative cause. The secondary endpoints were the changes in $\mathrm{SCr}$ and eGFR within $72 \mathrm{~h}$ after contrast agent exposure.

\section{Statistical analysis}

According to the study of Markota et al. [15], the sample size for the significance level of 0.05 , with the power of $90 \%$ is approximately 64 patients in each group. The categorical data were presented as number and percentages and the continuous data were expressed as means \pm standard deviation (SD). Comparison of continuous variables were analyzed by a Student's t-test and Paired t-test for normally distributed values or the Mann-Whitney U test and Wilcoxon rank sum test for non-normally distributed values. The categorical variables were compared using the $\chi^{2}$ test or Fisher's exact test if the expected frequency was $<5$. The one-way analysis of covariance (ANCOVA) was used for comparison of treatment effects between the two groups adjusting for baseline values and confound- ing factors. Statistical significance was defined as two-tailed $\mathrm{p}$ values less than 0.05 .

\section{Results}

The patients' baseline characteristics are presented in Table 1. There were no significant differences between the two groups in baseline clinical characteristics.

Before contrast exposure and $72 \mathrm{~h}$ thereafter, the changes in $\mathrm{SCr}$ and eGFR between the two groups were compared (Tables 2,3 ). In the nicorandil group, the median $\mathrm{SCr}$ concentration insignificantly increased from $1.08 \mathrm{mg} / \mathrm{dL}$ to $1.11 \mathrm{mg} / \mathrm{dL}$ at $72 \mathrm{~h}$ after radiocontrast administration ( $\mathrm{p}=0.088)$. In the control group, the median $\mathrm{SCr}$ concentration significantly increased from $1.03 \mathrm{mg} / \mathrm{dL}$ to $1.13 \mathrm{mg} / \mathrm{dL}$ at $72 \mathrm{~h}$ after radiocontrast administration $(\mathrm{p}<0.001)$.

In the nicorandil group, the mean eGFR insignificantly decreased from $76.39 \mathrm{~mL} / \mathrm{min} / 1.73 \mathrm{~m}^{2}$ to $73.96 \mathrm{~mL} / \mathrm{min} / 1.73 \mathrm{~m}^{2}$ at $72 \mathrm{~h}$ after radiocontrast administration $(\mathrm{p}=0.067)$. In the control group, the mean eGFR significantly decreased from $83 \mathrm{~mL} / \mathrm{min} / 1.73 \mathrm{~m}^{2}$ to $76.23 \mathrm{~mL} / \mathrm{min} / 1.73 \mathrm{~m}^{2}$ at $72 \mathrm{~h}$ after radiocontrast administration $(\mathrm{p}<0.001)$. 
Table 2. Biochemical and renal function changes before and $72 \mathrm{~h}$ after contrast medium exposure.

\begin{tabular}{lcccc}
\hline Groups & Measurement & Baseline & After intervention & P* \\
\hline Serum creatinine $[\mathrm{mg} / \mathrm{dL}]$ & Nicorandil group & $1.0859 \pm 0.22$ & $1.1125 \pm 0.19$ & 0.088 \\
& Saline group & $1.0359 \pm 0.15$ & $1.1344 \pm 0.18$ & $<0.001$ \\
& Nicorandil group & $76.39 \pm 24.6$ & $73.96 \pm 23.3$ & 0.067 \\
eGFR $\left[\mathrm{mL} / \mathrm{min} / 1.73 \mathrm{~m}^{2}\right]$ & Saline group & $83 \pm 28.1$ & $76.23 \pm 26.3$ & $<0.001$ \\
& & &
\end{tabular}

*Within-group comparisons were assessed using Paired t-test for normally distributed value or the Wilcoxon rank sum test for non-normally distributed values; eGFR — estimated glomerular filtration rate

Table 3. Results of ANCOVA adjusting for age and baseline values of dependent variables.

\begin{tabular}{lccccccc}
\hline Group & MD* & SE* & 95\% Cl for MD & Effect & Power & P* \\
& & & & \multicolumn{2}{c}{ size } & \\
\hline SCr (nicorandil vs. normal saline) & -0.059 & 0.022 & -0.103 & -0.015 & 0.254 & 0.802 & 0.009 \\
eGFR (nicorandil vs. normal saline) & 3.96 & 1.72 & 0.55 & 7.38 & 0.206 & 0.627 & 0.023 \\
\hline
\end{tabular}

$\mathrm{Cl}$ - confidence interval; eGFR - estimated glomerular filtration rate; MD - mean differences after intervention between two group; SCr serum creatinine; SE - standard error of mean; $p$ - obtained from ANCOVA after adjusting for age and baseline values of dependent variables

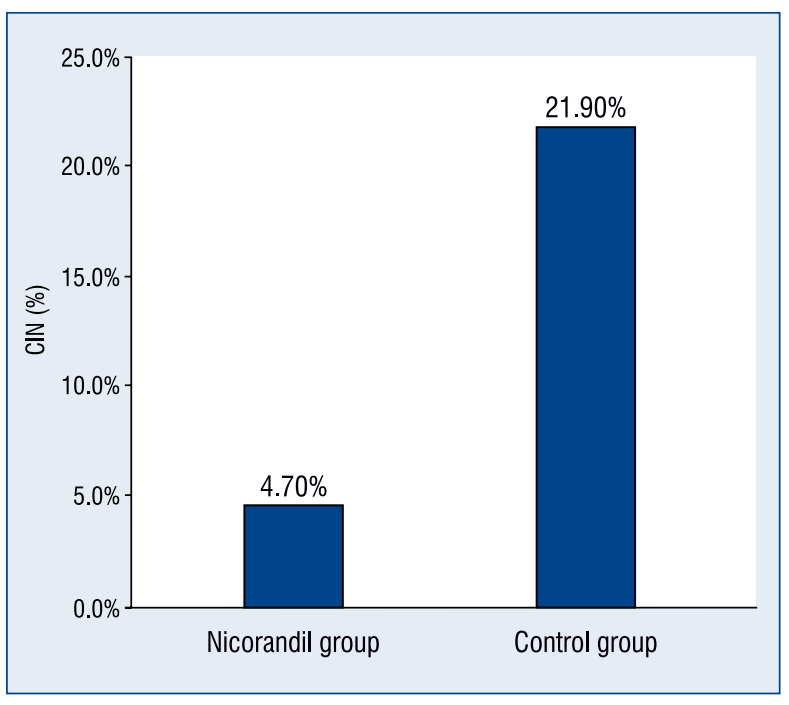

Figure 1. Incidence of contrast-induced nephropathy $(\mathrm{CIN})$ is significantly lower in the nicorandil group $(n=64)$ compared to the control group $(n=64) ; p=0.008$.

CIN occurred in 14 out of $64(21.9 \%)$ patients in the control group and in 3 out of 64 $(4.7 \%)$ patients in the nicorandil group. There was a significant difference in the incidence of CIN between the two groups at $72 \mathrm{~h}$ after administering the radiocontrast agent. The odds of CIN in the control group were 5.69 times higher than the intervention group ( $\mathrm{p}=0.008, \mathrm{CI} 95 \%$ for $\mathrm{OR}=$ $=1.54,20.92)$ (Fig. 1).

\section{Discussion}

In this study, the efficacy of oral nicorandil for the prevention from CIN was evaluated.

According to the available research, this study has shown for the second time that prophylactic oral administration of nicorandil had significant reduction in CIN incidence in high risk populations for the development of CIN. In addition, the main finding of the present study was that the use of low-dose oral nicorandil reduced CIN as well as having fewer side effects. In general, few studies have focused on the preventive effects of nicorandil on CIN. In the study by Ko et al. [12], 81 patients with an eGFR $<60 \mathrm{~mL} / \mathrm{min}$ received nicorandil $12 \mathrm{mg}$ intravenously for $30 \mathrm{~min}$ prior to coronary angiography. This study showed that prophylactic intravenous infusion of nicorandil did not decrease the incidence of CIN in patients with renal dysfunction undergoing coronary angiography [11]. In another study by Fan et al. [13], 120 patients with an eGFR of $60 \mathrm{~mL} / \mathrm{min}$ or less who were undergoing elective cardiac catheterization received nicorandil $10 \mathrm{mg}$ orally, three times daily from 2 days before to 3 days after the procedure. In that study, the incidence of CIN at $48 \mathrm{~h}$ after procedure was significantly lower in nicorandil group compared to the control group. This was in accordance with the present study that oral nicorandil decreases the incidence of CIN in high-risk patients undergoing elective cardiac catheterization. Various pharmacologic and therapeutic interventions have been 
applied to decrease the occurrence of CIN including $\mathrm{NAC}$, theophylline, calcium antagonists, diuretics, ascorbic acid, statins, sodium bicarbonate, adenosine antagonists, fenoldopam, atrial natriuretic peptide and other agents [3, 8]. Among them, NAC has been of considerable interest and several clinical studies and meta-analyses have been performed to assess the efficacy of NAC in the prevention of CIN. However, the NAC results have also been controversial and the optimal therapeutics remain unclear $[1,16,17]$. Currently, peri-procedural intravenous hydration, using iso-osmolar and/or lowosmolar contrast agents instead of high-osmolar agents and limiting the dose of contrast media are the confirmed strategies against CIN $[18,19]$. In this study, Iohexol (low-osmolar, non-ionic contrast medium) was also used to reduce complications and CIN incidence.

Although, the exact pathophysiology of CIN remains unclear, the possible direct and indirect pathophysiologic effects of contrast exposure that have been suggested include renal vasoconstriction, which leads to decreased oxygenation of the medulla causing ischemia and renal injury, direct tubular toxicity due to the creation of oxygen free radicals, causing acute tubular necrosis, and a decrease in glomerular filtration due to the alterations of tubulo-glumeral regulatory mechanisms $[4,8$, 20, 21]. Nicorandil, a K-ATP channel opener and a NO donor, is currently used in the treatment of angina and acute heart failure. Recent studies showed that activation of the K-ATP channel ameliorate ischemia-reperfusion in the kidney by preventing accumulation of reactive oxygen radicals (ROS) in mitochondria. In addition, these findings revealed that nicorandil might protect the kidney from the ischemic injury associated with the use of contrast media by inducing NO production and suppressing synthesis of endothelin-1 [11-13]. However, the exact mechanisms of the effect of nicorandil on CIN are unknown and further studies are required to assess the exact mechanism.

\section{Limitations of the study}

This study was faced with a series of limitations. First, the main limitation of the present study was that it was not a multi-centered, a double-blind study which may contain an observer bias requires verification. Second, the creatinine clearance was computed using the Cockcroft-Gault formula, rather than a direct measurement. Third, the sample size was small. A larger multi-centered, double-blind, and randomized trial including other clinical settings are required to confirm the beneficial effects of nicorandil on preventing CIN. Furthermore, this study is restricted by the lack of independent cost engineering committee (CEC).

The present findings revealed that oral nicorandil had substantial efficacy over hydration protocol in high-risk patients for the development of CIN. It is therefore concluded that the use of nicorandil could be effective in prevention of CIN; however, further controlled clinical studies are needed to resolve the uncertainties concerning the relative effectiveness of nicorandil in CIN prevention.

\section{Acknowledgements}

This study has been supported by the Deputy of Research and Education of Qom University of Medical Sciences. The authors would like to thank all the participants and private patients of Shahid Beheshti Hospital in city of Qom for their cooperation.

\section{Conflict of interest: None declared}

\section{References}

1. Thayssen P, Lassen JF, Jensen SE, et al. Prevention of contrast-induced nephropathy with $\mathrm{N}$-acetylcysteine or sodium bicarbonate in patients with ST-segment-myocardial infarction: a prospective, randomized, open-labeled trial. Circ Cardiovasc Interv. 2014; 7(2): 216-224, doi: 10.1161/CIRCINTERVENTIONS.113.000653, indexed in Pubmed: 24714489.

2. Cheungpasitporn W, Thongprayoon C, Brabec BA, et al. Oral hydration for prevention of contrast-induced acute kidney injury in elective radiological procedures: a systematic review and meta-analysis of randomized controlled trials. N Am J Med Sci. 2014; 6(12): 618-624, doi: 10.4103/1947-2714.147977, indexed in Pubmed: 25599049.

3. Wu MY, Hsiang HF, Wong CS, et al. The effectiveness of $\mathrm{N}$-Acetylcysteine in preventing contrast-induced nephropathy in patients undergoing contrast-enhanced computed tomography: a meta-analysis of randomized controlled trials. Int Urol Nephrol. 2013; 45(5): 1309-1318, doi: 10.1007/s11255-012-0363-1, indexed in Pubmed: 23283594.

4. Chyou AC, Thodge A, Feldman DN, et al. Statins in the prevention of contrast-induced nephropathy. Curr Treat Options Cardiovasc Med. 2015; 17(4): 375, doi: 10.1007/s11936-015-0375-0, indexed in Pubmed: 25778426.

5. Wong PC, Li Z, Guo J, et al. Pathophysiology of contrast-induced nephropathy. Int J Cardiol. 2012; 158(2): 186-192, doi: 10.1016/j. ijcard.2011.06.115, indexed in Pubmed: 21784541.

6. Wu Z, Zhang H, Jin W, et al. The effect of renin-angiotensinaldosterone system blockade medications on contrast-induced nephropathy in patients undergoing coronary angiography: a meta-analysis. PLoS One. 2015; 10(6): e0129747, doi: 10.1371/ /journal.pone.0129747, indexed in Pubmed: 26083525.

7. Wessely R, Koppara T, Bradaric C, et al. Contrast Media and Nephrotoxicity Following Coronary Revascularization by Angioplasty Trial Investigators. Choice of contrast medium in patients 
with impaired renal function undergoing percutaneous coronary intervention. Circ Cardiovasc Interv. 2009; 2(5): 430-437, doi: 10.1161/CIRCINTERVENTIONS.109.874933, indexed in Pubmed: 20031753.

8. Kassis HM, Minsinger KD, McCullough PA, et al. A Review of the use of iloprost, a synthetic prostacyclin, in the prevention of radiocontrast nephropathy in patients undergoing coronary angiography and intervention. Clin Cardiol. 2015; 38(8): 492-498, doi: 10.1002/clc.22407, indexed in Pubmed: 25963191.

9. Erol T, Tekin A, Katırcıbaşı MT, et al. Efficacy of allopurinol pretreatment for prevention of contrast-induced nephropathy: a randomized controlled trial. Int J Cardiol. 2013; 167(4): 1396-1399, doi: 10.1016/j.ijcard.2012.04.068, indexed in Pubmed: 22572633

10. Çiçek G, Bozbay M, Açıkgöz SK, et al. The ratio of contrast volume to glomerular filtration rate predicts in-hospital and six-month mortality in patients undergoing primary angioplasty for ST-elevation myocardial infarction. Cardiol J. 2015; 22(1): 101-107, doi: 10.5603/CJ.a2014.0027, indexed in Pubmed: 24671903.

11. Shimizu S, Saito M, Kinoshita Y, et al. Nicorandil ameliorates ischaemia-reperfusion injury in the rat kidney. Br J Pharmacol. 2011; 163(2): 272-282, doi: 10.1111/j.1476-5381.2011.01231.x, indexed in Pubmed: 21250976.

12. Ko YG, Lee BK, Kang WC, et al. PRINCIPLE Investigators. Preventive effect of pretreatment with intravenous nicorandil on contrast-induced nephropathy in patients with renal dysfunction undergoing coronary angiography (PRINCIPLE Study). Yonsei Med J. 2013; 54(4): 957-964, doi: 10.3349/ymj.2013.54.4.957, indexed in Pubmed: 23709432.

13. Fan Y, Wei Q, Cai J, et al. Preventive effect of oral nicorandil on contrast-induced nephropathy in patients with renal insufficiency undergoing elective cardiac catheterization. Heart Vessels. 2016; 31(11): 1776-1782, doi: 10.1007/s00380-016-0809-y, indexed in Pubmed: 26874946.

14. Mehran R, Aymong ED, Nikolsky E, et al. A simple risk score for prediction of contrast-induced nephropathy after percuta- neous coronary intervention: development and initial validation. J Am Coll Cardiol. 2004; 44(7): 1393-1399, doi: 10.1016/j. jacc.2004.06.068, indexed in Pubmed: 15464318.

15. Markota D, Markota I, Starcevic B, et al. Prevention of contrast-induced nephropathy with $\mathrm{Na} / \mathrm{K}$ citrate. Eur Heart J. 2013; 34(30): 2362-2367, doi: 10.1093/eurheartj/eht009, indexed in Pubmed: 23349296.

16. O'Sullivan S, Healy DA, Moloney MC, et al. The role of Nacetylcysteine in the prevention of contrast-induced nephropathy in patients undergoing peripheral angiography: a structured review and meta-analysis. Angiology. 2013; 64(8): 576-582, doi: 10.1177/0003319712467223, indexed in Pubmed: 23188834.

17. Wu MY, Hsiang HF, Wong CS, et al. The effectiveness of $\mathrm{N}$-acetylcysteine in preventing contrast-induced nephropathy in patients undergoing contrast-enhanced computed tomography: a meta-analysis of randomized controlled trials. Int Urol Nephrol. 2013; 45(5): 1309-1318, doi: 10.1007/s11255-012-0363-1, indexed in Pubmed: 23283594.

18. Gomes VO, Poli de Figueredo CE, Caramori P, et al. N-acetylcysteine does not prevent contrast induced nephropathy after cardiac catheterisation with an ionic low osmolality contrast medium: a multicentre clinical trial. Heart. 2005; 91(6): 774-778, doi: 10.1136/hrt.2004.039636, indexed in Pubmed: 15894775.

19. Gu G, Zhang Y, Lu R, et al. Additional furosemide treatment beyond saline hydration for the prevention of contrast-induced nephropathy: a meta-analysis of randomized controlled trials. Int J Clin Exp Med. 2015; 8(1): 387-394, indexed in Pubmed: 25785009.

20. Xing $\mathrm{Y}$, Wei $\mathrm{Rb}$, Tang $\mathrm{Lu}$, et al. Protective effect of salidroside on contrast-induced nephropathy in comparison with $\mathrm{N}$-acetylcysteine and its underlying mechanism. Chin J Integr Med. 2015; 21(4): 266-273, doi: 10.1007/s11655-015-2137-y, indexed in Pubmed: 25877653.

21. Tumlin J, Stacul F, Adam A, et al. CIN Consensus Working Panel. Pathophysiology of contrast-induced nephropathy. Am J Cardiol. 2006; 98(6A): 14K-20K, doi: 10.1016/j.amjcard.2006.01.020, indexed in Pubmed: 16949376. 